OPEN ACCESS

Edited by:

Laurent Pierre Nicod,

University of Lausanne, Switzerland

Reviewed by:

Martin Petrek,

Palacký University, Olomouc, Czechia

Vincent Aubert,

Centre Hospitalier Universitaire

Vaudois (CHUV), Switzerland

*Correspondence:

Antoine Roux

a.roux@hopital-foch.org

Specialty section:

This article was submitted

to Pulmonary Medicine,

a section of the journal

Frontiers in Medicine

Received: 10 July 2017

Accepted: 08 September 2017

Published: 11 October 2017

Citation:

Roux A, Bendib Le Lan I, Holifanjaniaina S, Thomas $K A$, Picard C, Grenet D, De Miranda S,

Douvry B, Beaumont-Azuar L,

Sage E, Devaquet J, Cuquemelle E,

Le Guen M, Suberbielle C

Gautreau C, Stern M, Rossetti M,

Hamid AM and Parquin F (2017)

Characteristics of Donor-Specific

Antibodies Associated With

Antibody-Mediated Rejection

in Lung Transplantation.

Front. Med. 4:155

doi: 10.3389/fmed.2017.00155

\section{Characteristics of Donor-Specific Antibodies Associated With Antibody-Mediated Rejection in Lung Transplantation}

\author{
Antoine Roux ${ }^{1,2,3 *}$, Ines Bendib Le Lan', Sonia Holifanjaniaina ${ }^{4}$, Kimberly A. Thomas ${ }^{2}$, \\ Clément Picard', Dominique Grenet ${ }^{1}$, Sandra De Miranda', Benoit Douvry', \\ Laurence Beaumont-Azuar ${ }^{1}$, Edouard Sage ${ }^{3,5}$, Jérôme Devaquet ${ }^{6}$, Elise Cuquemelle ${ }^{7}$, \\ Morgan Le Guen ${ }^{8}$, Caroline Suberbielle 9 , Chantal Gautreau ${ }^{9}$, Marc Stern $^{1}$, \\ Maura Rossetti ${ }^{2}$, Abdul Monem Hamid ${ }^{1}$ and Francois Parquin ${ }^{6}$ on behalf of the \\ Foch Lung Transplantation Group
}

${ }^{1}$ Pneumology, Adult CF Center, Lung Transplantation Department, Foch Hospital, Suresnes, France, ${ }^{2}$ Department of Pathology and Laboratory Medicine, University of California, Los Angeles, Los Angeles, CA, United States, ${ }^{3}$ Université Versailles Saint-Quentin-en-Yvelines, Montigny le Bretonneux, France, ${ }^{4}$ Pathology Department, Foch Hospital, Suresnes, France, ${ }^{5}$ Thoracic Surgery Department, Foch Hospital, Suresnes, France, ${ }^{6}$ Intensive Care Unit, Foch Hospital, Suresnes, France, ${ }^{7}$ Thoracic Intensive Care Unit, Foch Hospital, Suresnes, France, ${ }^{8}$ Anesthesiology Department, Foch Hospital, Suresnes, France, ${ }^{9}$ Laboratoire Régional d'Histocompatibilité, Saint-Louis Hospital, Assistance Publique-Hôpitaux de Paris, Paris, France

Although donor-specific anti-human leukocyte antigen (HLA) antibodies (DSAs) are frequently found in recipients after lung transplantation (LT), the characteristics of DSA which influence antibody-mediated rejection (AMR) in LT are not fully defined. We retrospectively analyzed 206 consecutive LT patients of our center (2010-2013). DSAs were detected by using luminex single antigen beads assay and mean fluorescence intensity was assessed. Within the study population, 105 patients had positive DSA. Patients with and without AMR (AMR ${ }^{\text {Pos }}, n=22$, and $\mathrm{AMR}^{\mathrm{Neg}}, n=83$, respectively) were compared. $\mathrm{AMR}^{\mathrm{Pos}}$ patients had significantly greater frequencies of anti-HLA DQ DSA (DQ DSA) than $\mathrm{AMR}^{\mathrm{Neg}}$ patients (95 vs 58\%, respectively, $p<0.0001$ ). Compared to AMR $^{\text {Neg }}$ patients, AMR $^{\text {Pos }}$ patients had higher DQ DSA sum MFI $[7,332(2,067-10,213)$ vs $681(0-1,887), p<0.0001]$. DQ DSA when associated with AMR, had more frequent graft loss and chronic lung allograft dysfunction (CLAD). These data suggest (i) that DSA characteristics clearly differ between $\mathrm{AMR}^{\text {Pos }}$ and $\mathrm{AMR}^{\mathrm{Neg}}$ patients and (ii) the deleterious impact of DQ DSA on clinical outcome.

Keywords: HLA, donor-specific antibodies, lung transplant, clinical outcome, antibody mediated rejection

\section{INTRODUCTION}

The role of donor-specific anti-human leukocyte antigen (HLA) antibody (DSA) in graft failure via antibody-mediated rejection (AMR) and sub-clinical chronic AMR has been widely established in kidney transplantation (1-3) and heart transplantation (4) (KT and HT, respectively). Our group (5) and others $(6,7)$ demonstrated in the context of lung transplantation (LT) that AMR was associated

Abbreviations: Ab, antibody; AMR, antibody-mediated rejection; CLAD, chronic lung allograft dysfunction; DSA, donorspecific antibody; DQ DSA, anti-HLA DQ donor-specific antibody; Non DQ DSA, DSA without reactivity against HLA DQ antigen; FEV1, forced expiratory volume in 1 second; HT, heart transplantation; KT, kidney transplantation; LT, lung transplantation; MFI, mean fluorescence intensity; SAB, single antigen beads. 
with chronic lung allograft dysfunction (CLAD) and poor graft survival. Previous studies concerning the prognostic value of DSA, despite the significant contribution of knowledge regarding DSA in solid organ transplantation (SOT), provide only limited characterization of DSA in the setting of LT. As a matter of fact, solid phase assay as luminex single antigen beads (SAB) assay cannot be used for quantization of the DSA strength but allow determination of appropriate mean fluorescence intensity (MFI) threshold for DSA identification or their impact on clinical status.

Two retrospective analyses of KT cohorts $(8,9)$ have shown that presence of pre-transplant (preTx) DSA and MFI of the immunodominant DSA (understood as the DSA with the highest MFI for a given patient) were associated with graft loss. These publications did not further describe DSA either post-transplant (postTx) or at the moment of AMR. Comparison of DSA MFI from $\mathrm{SAB}$ tests and from a complement binding test suggested that the MFI of immunodominant DSA or the sum MFI of all DSA may be as efficient as the complement binding test for AMR prediction and graft failure (10). More recently, Tikkanen et al. showed that particularly HLA DQ mismatch and subsequent DQ DSA were associated with CLAD, yet the results did not show if the MFI was also associated with poor graft outcome (11). Moreover, these studies did not integrate potential AMR occurrence associated with DQ DSA for the analysis of graft outcome.

In this study, we propose an extended analysis of DSA characteristics in our cohort previously described for AMR impact on graft prognosis (5). We took advantage of our extensive DSA monitoring strategy and prospective assessment of AMR diagnosis to analyze DSA characteristics according to AMR status and thereby evaluate their diagnostic performance and evaluate the clinical outcomes associated with DQ DSA.

\section{MATERIALS AND METHODS}

\section{Patient Population, DSA Monitoring Strategy, and HLA Testing}

All consecutive patients of the lung transplant cohort in Foch Hospital from January 2010 to December 2013 were eligible. Patients were routinely tested for HLA antibody (HLA-Ab) postTx at days 1, 7, 21, and 30; at months 2, 3, 4, 6, 9, and 12; and then every 6 months thereafter. From January 2010 to December 2012, patients were tested by first tested by LabScreen Mixed ${ }^{\circledR}$ (LSM, One Lambda) at these scheduled time point. At least once in the first 3 months and at month 12, and if positivity of Labscreen Mixed or graft failure, serum were further tested with LabScreen Single Antigen ${ }^{\circledR}$ (LSA, One Lambda, Canoga Park, CA, USA). After December 2012, patients were systematically tested by LSA at each time point. In our analysis, negative results of either LSM or LSA were reported as negative for DSA. Patient's HLA typing was done using standard molecular biology techniques (SSO, One Lambda ${ }^{\circledR}$ ) and then reported as serological equivalents in clinical reports. The One Lambda kits were used according to manufacturer's recommendations. Deceased donor's HLA typing was performed by serological typing and/or molecular biology (PCR-SSP) according to the European Federation of Immunogenetics rules.
Donor-specific antibody positivity was defined if the beads loaded with donor HLA antigen specificity had MFI >500. Specificity is assigned considering the highest MFI bead when several beads express the same antigen.

\section{Analysis of DSA Characteristics}

The immunodominant DSA was defined as the DSA with highest MFI in a given serum sample. DQ DSA specificities were reported for HLA-DQB only. The peak was defined as the time point with the highest sum MFI for $\mathrm{AMR}^{\mathrm{Neg}}$ patients or the time point of AMR diagnosis for $\mathrm{AMR}^{\mathrm{Pos}}$ patients. At the peak, we compared the number of DSA specificities, the MFI of the immunodominant, Class I, Class II, DQ DSA, preformed, and de novo DSA, and the sum MFI between $\mathrm{AMR}^{\mathrm{Pos}}$ and $\mathrm{AMR}^{\mathrm{Neg}}$ patients.

\section{AMR Categorization}

Antibody-mediated rejection categorization was established prospectively, as described in our previous publication (5), by a multidisciplinary physician and pathologist team using the assessments listed below. All AMR cases met criteria of ISHLT consensus for definite or probable DSA positive AMR (12). Briefly, patients with AMR $\left(A M R^{\text {Pos }}\right)$ were defined by three criteria: (i) the presence of HLA DSA (DSA ${ }^{\text {Pos: }}$ DSA MFI >1,000, or MFI = 500-1,000 with more than two specificities and/or detected more than once), (ii) biopsy patterns relative to AMR [including positive C4d staining and/or histological pattern compatible with AMR (i.e., neutrophil capillaritis, or acute lung injury with or without organizing pneumonia)], and (iii) graft failure ( $-20 \%$ decrease in forced expiratory volume in 1 second or hematosis degradation requiring introduction/increase of oxygenotherapy or mechanical ventilation). $D_{S A}{ }^{\text {Pos }}$ patients without graft failure and biopsy patterns indicative of AMR were defined as $\mathrm{AMR}^{\mathrm{Neg}}$ (Table 1).

\section{Clinical Outcome}

For clinical outcome analysis we used (i) graft survival conditioned by 3-month survival, defined as graft survival among patients alive at month 3 allowing enough followup to consider the absence of DSA or DQ DSA as meaningful (ii) freedom from CLAD (chronic lung allograft dysfunction) as defined previously (13) within patients alive at 6 months and excluding patient with bronchial issue as previously reported (5).

\section{Ethics}

This observational study was approved by the research protocol evaluation committee of the Institutional Review Board of the French Learned Society for Respiratory Medicine-Société de Pneumologie de Langue Française. Every patient in this study was enrolled in a prospective non-therapeutic interventional research protocol[eitherCOLT(NCT00980967,ID-RCB:2009-A00036-51) or RhumTP (NCT01791166, ID-RCB:2010-A01174-35)]. Upon enrollment, all patients signed consent for research use of their clinical data.

\section{Statistical Analysis}

Categorical variables were expressed as number and percentage and compared by Chi- square test or Fisher's exact test as appropriate. Quantitative variables were expressed as median and 
interquartile 25-75 (IQR 25-75) or mean and 95\% confidence interval (CI 95\%) or SD and compared by Mann-Whitney or paired Wilcoxon signed-rank test for repeated measures. Area under the curve (AUC), specificity, and sensitivity of MFI for AMR diagnosis were determined using the ROC method. Survival analysis was calculated by log-rank test.

All analyses were performed using Prism ${ }^{\circledR}$ v5.0 for Mac OS X (Graphpad Software, San Diego, CA, USA). A p-value below 0.05 was considered statistically significant.

\section{RESULTS}

\section{Study Population}

Among 209 eligible patients, 206 were included in the analysis. Of these 206, 88 patients had no detectable DSA during the entire followup period (DSA ${ }^{\mathrm{Neg}}$ ), 13 had one DSA once with an MFI
500-1,000 (DSA ${ }^{\text {Lim }}$ ), and 105 patients were DSA ${ }^{\text {Pos }}$. Within the $D_{S A}{ }^{\text {Pos }}$ group, 22 patients were prospectively diagnosed with AMR $\left(\mathrm{AMR}^{\mathrm{Pos}}\right)$, while 83 had at least one positive DSA detection during the whole followup but no AMR diagnosis $\left(\mathrm{AMR}^{\mathrm{Neg}}\right)$ (Figure 1). $\mathrm{AMR}^{\mathrm{Pos}}$ and $\mathrm{AMR}^{\mathrm{Neg}}$ patients did not differ for clinical baseline characteristics except for the number of HLA mismatches, which was higher in $\mathrm{AMR}^{\mathrm{Pos}}$ patients (Table 2). Interestingly, the frequency of patients having two DQ mismatches significantly differed between $\mathrm{AMR}^{\text {Pos }}(68 \%)$ and $\mathrm{AMR}^{\mathrm{Neg}}$ patients $(26.5 \%)$ but the frequency of presensitized patients did not differ between the two group (12 (54\%) vs. 58 (69\%), respectively).

\section{Immunodominance As an Indicator of AMR}

The immunodominant DSA MFI at the peak time point was significantly higher for $\mathrm{AMR}^{\mathrm{Pos}}$ than for $\mathrm{AMR}^{\mathrm{Neg}}$ patients (Figure 2A).

TABLE 1 | Criteria for antibody-mediated rejection-donor-specific antibody status categorization.

Antibody-mediated rejection (AMR) patients (DSA ${ }^{\text {pos }} \mathrm{AMR}^{\text {pos }}$ )

\begin{tabular}{lll}
\multicolumn{3}{c}{ Non-AMR patients } \\
\hline AMR $^{\text {neg }}$ (DSA $^{\text {pos }}$ AMR $^{\text {neg }}$ ) & DSA $^{\text {Lim }}$ & DSA $^{\text {neg }}$ \\
DSA positivity (DSA MFI $>1,000$, & DSA detected & All single \\
or MFI = 500-1,000 with more & only once and & antigen tests \\
than two specificities, and/or & having only one & with DSA \\
detected more than once) & specificity with an & MFI $<500$
\end{tabular}

Donor-specific antibody (DSA) positivity [DSA mean fluorescence

intensity (MFI) $>1,000$, or MFI $=500-1,000$ with more than two

specificities, and/or detected more than once]

\begin{tabular}{|c|c|}
\hline AMR C4dpos & AMR C4dneg \\
\hline $\begin{array}{l}\text { Clinical dysfunction and DSA positivity and C4d positive } \\
\text { staining with or without histological patterns suggestive of AMR }\end{array}$ & $\begin{array}{l}\text { Clinical dysfunction } \\
\text { and DSA positivity and } \\
\text { negative C4d staining } \\
\text { with histological patterns } \\
\text { suggestive of AMR: } \\
\text { neutrophil capillaritis }{ }^{\mathrm{a}} \text {; } \\
\text { acute lung injury }^{\mathrm{a}}\end{array}$ \\
\hline
\end{tabular}

${ }^{a}$ At the exclusion of other diagnoses (ischemia-reperfusion, infection, aspiration, and drug toxicity).

No AMR diagnosis through entire follow up

\section{Analysis of DSA status at each time point}

Temporal DSA/AMR Categorization

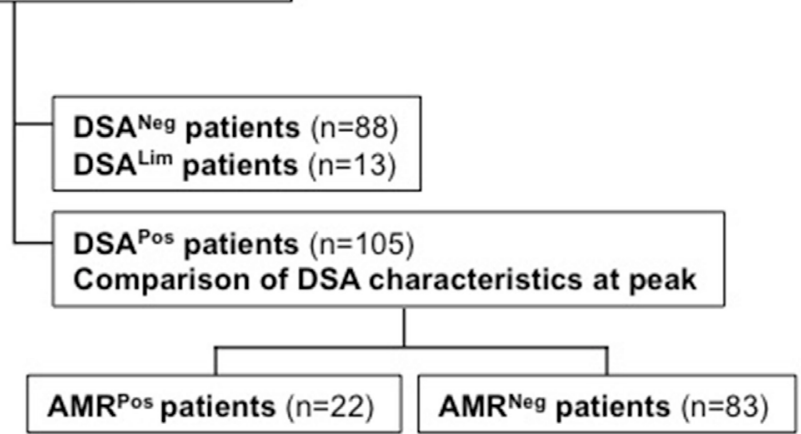

FIGURE 1 | Flow chart and analysis summary. 
TABLE 2 | Baseline clinical characteristics.

\begin{tabular}{lccc}
\hline & AMR $^{\text {Pos }}$ patients & AMR $^{\text {Neg }}$ patients & $p$ \\
\hline $\begin{array}{l}\text { Recipient age, median } \\
\text { (IQR 25-75) }\end{array}$ & $35.45(23.65-53.25)$ & $36.30(27.7-51.3)$ & 0.78 \\
$\begin{array}{l}\text { Underlying disease } \\
\text { (CF/PF/COPD/other), } n\end{array}$ & $10 / 5 / 4 / 3$ & $45 / 15 / 15 / 8$ & 0.86 \\
Recipient female, $n$ (\%) & $11(50)$ & & \\
LAS, median (IQR 25-75) & $36.85(33.2-42.1)$ & $38(35.1-43.7)$ & 0.44 \\
HLA mismatch, mean (SD) & $6.9(0.7)$ & $5.9(1.2)$ & $<0.001$ \\
PreLT DSA, $n$ (\%) & $12(54)$ & $58(69)$ & 0.2 \\
Induction therapy, $n$ (\%) & $12(54)$ & $52(62.3)$ & 0.62 \\
\hline
\end{tabular}

Categorical variables were compared by Fisher's exact test. Quantitative variables were compared by Mann-Whitney test. HLA mismatching was defined as the number of donor HLA antigens among loci $A, B, D R B 1, D Q B 1$ differing from recipient antigens ( 4 loci $\times 2$ alleles each $=$ maximum of 8 mismatches).

CF, cystic fibrosis; COPD, chronic obstructive pulmonary disease; LAS, lung allocation score; PF, pulmonary fibrosis.
Given the significant difference and high deviation for either median or mean values between $\mathrm{AMR}^{\mathrm{Pos}}$ and $\mathrm{AMR}^{\mathrm{Neg}}$, we evaluated the accuracy of using the immunodominant DSA MFI for AMR diagnosis. The AUC was 0.84, with an estimated sensitivity of $95.45 \%$ with a low cutoff $(2,100)$ and specificity of $100 \%$ with an upper cutoff $(13,061)$ (Figure 2B). The seven $\mathrm{AMR}^{\mathrm{Pos}}$ patients with immunodominant MFI < 5,000 are more precisely described in Figure 2C: all patients had DSA associated with C4d + AMR; all except one had DQ dominant DSA; and 4 out of 7 had greater than two different specificities. Of note sera with low MFI from $\mathrm{AMR}^{\text {Pos }}$ patients were all retested with EDTA and showed similar MFI excluding prozone effect for those patients.

\section{Sum MFI As an Indicator of AMR}

Despite the use of immunodominant DSA as an important indicator of AMR status, that particular DSA characteristic did
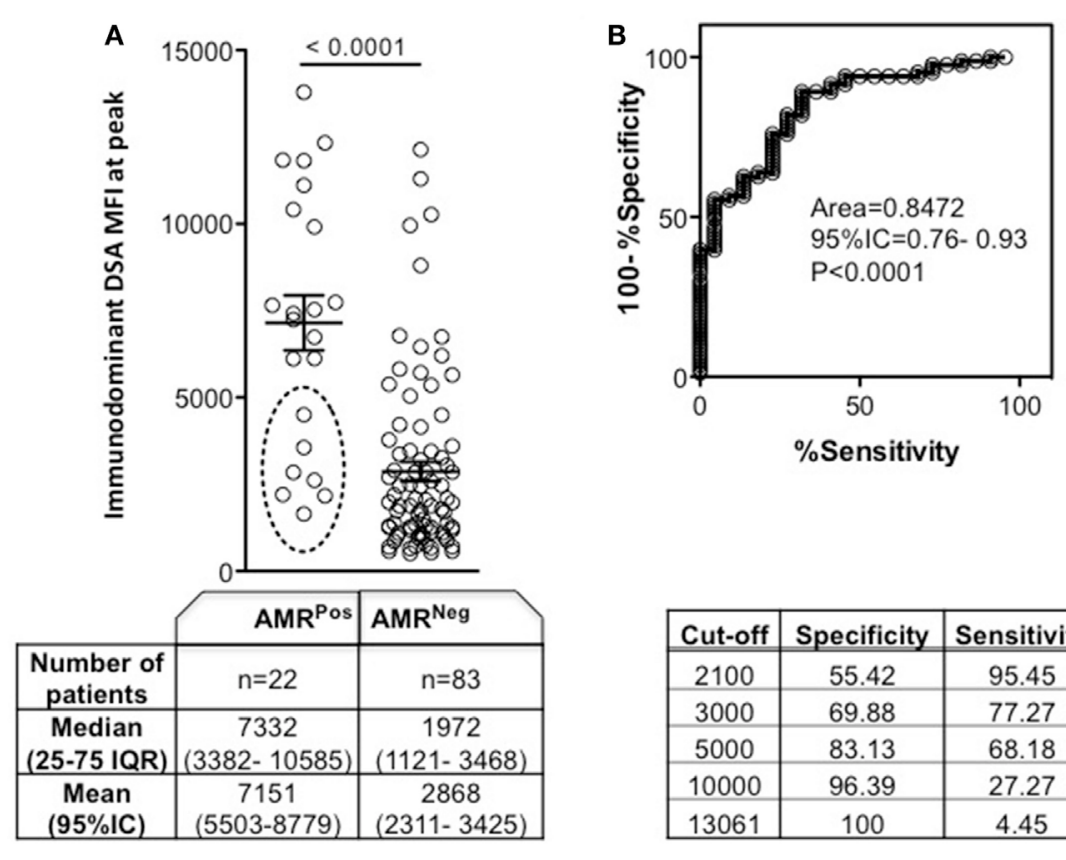

C

\begin{tabular}{|c|c|c|}
\hline Cut-off & Specificity & Sensitivity \\
\hline 2100 & 55.42 & 95.45 \\
\hline 3000 & 69.88 & 77.27 \\
\hline 5000 & 83.13 & 68.18 \\
\hline 10000 & 96.39 & 27.27 \\
\hline 13061 & 100 & 4.45 \\
\hline
\end{tabular}

\begin{tabular}{|l|c|c|c|c|c|c|c|}
\hline Patient & $\begin{array}{c}\text { Delay } \\
\text { post LT }\end{array}$ & $\begin{array}{c}\text { C4d } \\
\text { staining } \\
\text { on TBB }\end{array}$ & $\begin{array}{c}\text { Number of } \\
\text { specificities }\end{array}$ & $\begin{array}{c}\text { Sum MFI } \\
\text { at AMR }\end{array}$ & $\begin{array}{c}\text { Higher MFI } \\
\text { at AMR }\end{array}$ & $\begin{array}{c}\text { DQ MFI } \\
\text { at AMR }\end{array}$ & $\begin{array}{c}\text { Specificity of } \\
\text { Immunodominant } \\
\text { DSA }\end{array}$ \\
\hline \hline Patient \#8 & 20 & + & 6 & 8037 & 2847 & 2847 & DQ7 \\
\hline Patient \#9 & 20 & + & 7 & 8030 & 2615 & 2615 & DQ7 \\
\hline Patient \#16 & 116 & + & 2 & 2613 & 1646 & 1646 & DQ7 \\
\hline Patient \#2 & 444 & + & 1 & 2174 & 2174 & 0 & A2 \\
\hline Patient \#22 & 10 & + & 1 & 2207 & 2207 & 2207 & DQ6 \\
\hline Patient \#3 & 128 & + & 2 & 4084 & 3562 & 4084 & DQ6 \\
\hline Patient \#4 & 21 & + & 1 & 4504 & 4504 & 4504 & DQ9 \\
\hline
\end{tabular}

FIGURE 2 | Mean fluorescence intensity (MFI) of immunodominant donor-specific antibody (DSA) at peak according to antibody-mediated rejection (AMR) status.

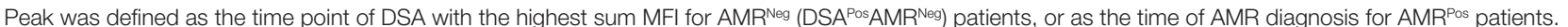
(A) Each patient's immunodominant DSA is a circle. Circles inside broken line ellipse $(n=7)$ represent patients with AMR despite an immunodominant DSA $\mathrm{MFI}<5,000$, whose characteristics are detailed in panel (C) (Mann-Whitney test). (B) ROC curve for use of immunodominant DSA MFI as a predictor of AMR diagnosis, and the specificity/sensitivity according to different MFI cut-offs. (C) Characteristics of the 7 AMRPos patients with immunodominant DSA MFI $<5,000$. Significance values are denoted above lines over compared groups. 
not provide information regarding the number of DSA specificities. Compared to $\mathrm{AMR}^{\mathrm{Neg}}, \mathrm{AMR}^{\text {Pos }}$ patients had significantly increased number of DSA specificities $(3.4 \pm 2.28$ vs $1.8 \pm 1.2$, $p=0.0013$ ) (Figure 3A). As this dimension has been shown to contribute to antibody pathogenicity (9), we then analyzed the sum MFI for each patient at the peak time point to account for the total number of specificities. The sum MFI of $\mathrm{AMR}^{\mathrm{Pos}}$ patients was significantly higher than the sum MFI of $A_{M R}{ }^{N e g}$ patients (Figure 3A). Similar to what was seen above with immunodominant MFI, sum MFI showed high diagnostic performance for AMR with $100 \%$ sensitivity at a lower cutoff $(2,100)$ and more than $97 \%$ specificity at an upper cutoff $(15,000)$ (Figure 3B). This assessment of sum MFI, regardless of DSA specificity or preformed/de novo nature, provides an interesting diagnostic performance for AMR diagnosis.

\section{DQ DSA As an Indicator of AMR and As a Determinant of Outcome}

As DQ specificity has been previously described (14) as a characteristic which correlates with AMR, we looked for an association of DQ DSA with AMR in our cohort. All AMR ${ }^{\text {Pos }}$ patients had DQ DSA except one (95\%) who was matched with donor
DQ, compared to only $58 \%$ of $\mathrm{AMR}^{\mathrm{Neg}}$ patients $(p<0.0001)$ (Figure 4A). The immunodominant DSA was specific for DQ in $18 / 22(81 \%) \mathrm{AMR}^{\text {Pos }}$ patients, but only in $30 / 83(36 \%) \mathrm{AMR}^{\mathrm{Neg}}$ patients $\left(p<0.0001\right.$, Figure 4B). For the four remaining $\mathrm{AMR}^{\mathrm{Pos}}$ patients, the immunodominant specificities were A2, Cw05, and DR13 (twice). The MFI of DQ DSA was higher in $\mathrm{AMR}^{\text {Pos }}$ patients (Figure 4C) and the diagnostic performance of DQ DSA MFI for AMR was similar to those of both the immunodominant and the sum MFI (Figure 4D).

Given the previously shown negative impact of DQ DSA on lung transplant outcome (11), we evaluate the association of DQ DSA with graft survival conditioned by the 3 months survival and CLAD occurrence. Compared to DSA negative patients and nonDQ DSA patients, DQ DSA patients had significantly worst graft survival conditioned by the 3 months survival and more frequent CLAD occurrence (Figures 5A,B). Of note, this comparison did not reach significance when comparing only non DQ DSA and DQ DSA patients.

Finally and more importantly, by splitting patients according to their DQ DSA and AMR status, the analysis showed a clearly worst outcome within DQ DSA AMR ${ }^{\text {pos }}$ patients than patients both DQ and non DQ AMR ${ }^{\mathrm{Neg}}$ patients (Figure 5C).
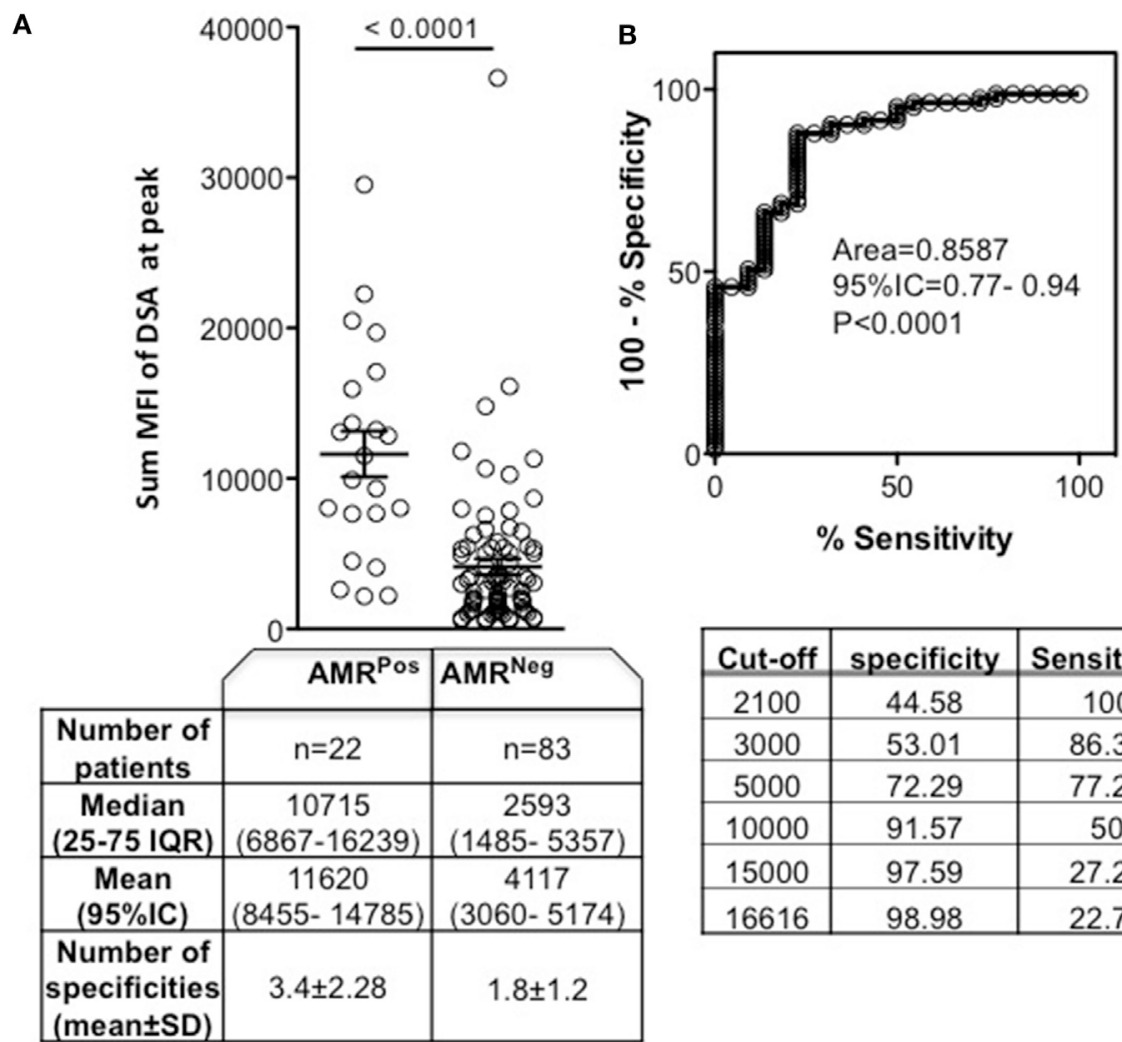

\begin{tabular}{|c|c|c|}
\hline Cut-off & specificity & Sensitivity \\
\hline 2100 & 44.58 & 100 \\
\hline 3000 & 53.01 & 86.36 \\
\hline 5000 & 72.29 & 77.27 \\
\hline 10000 & 91.57 & 50 \\
\hline 15000 & 97.59 & 27.27 \\
\hline 16616 & 98.98 & 22.73 \\
\hline
\end{tabular}

FIGURE 3 | Sum mean fluorescence intensity (MFI) of donor specific antibody (DSA) at peak according to antibody-mediated rejection (AMR) status. Peak was defined as the time point of DSA with the highest sum MFI for AMR ${ }^{\text {Neg }}$ (DSA ${ }^{\text {Pos } A M R ~}{ }^{\text {Neg }}$ ) patients, or as the time of AMR diagnosis for AMR ${ }^{\text {Pos }}$ patients. (A) The sum MFI of each patient's DSA is indicated by each circle. The table below lists the number of patients, median and mean sum MFI, and number of specificities for each patient group. (B) ROC curve for use of sum MFI as a predictor of AMR diagnosis and the specificity/sensitivity according to MFI different cutoffs. Significance values are denoted above lines over compared groups. 

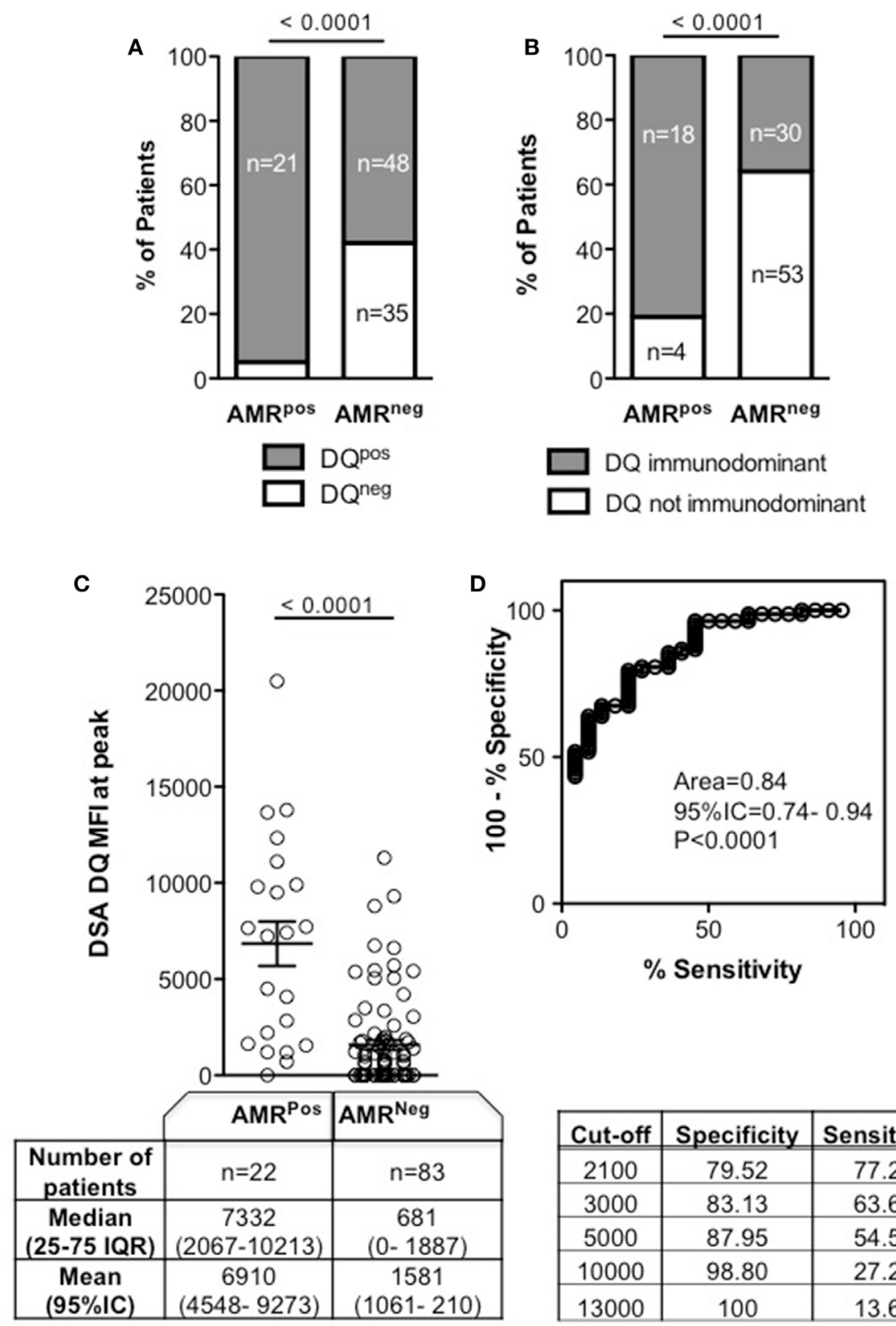

FIGURE 4 | DQ mean fluorescence intensity (MFI) of donor specific antibody (DSA) at peak according to antibody-mediated rejection (AMR) status. Peak was defined as the time point of DSA with the highest sum MFI for AMR ${ }^{\text {Neg }}$ (DSA ${ }^{\text {Pos } A M R}{ }^{\text {Neg }}$ ) patients, or as the time of AMR diagnosis for AMR ${ }^{\text {Pos }}$ patients. (A) Frequency and number of patients with DQ DSA at the peak. (B) Frequency and number of patients with immunodominant DQ DSA at the peak. (C) Each patient's DQ DSA $\mathrm{MFI}$ are represented as a circle. Comparison of number of patients, as well as median and mean DQ DSA MFI, for AMR ${ }^{\text {Pos }}$ and $A M R^{\text {Neg }}$ patients is in the table below. (D) ROC curve for use of DQ DSA MFI as a predictor of AMR diagnosis and the specificity/sensitivity according to different MFI cutoffs. Groups were compared using Chi-square $(\mathbf{A}, \mathbf{B})$ and Mann-Whitney tests $\mathbf{( C )}$ and significance values are denoted above lines over compared groups.

\section{DISCUSSION}

Our extensive analysis provides a global overview of DSA frequency after LT and shows a multidimensional discrepancy of DSA characteristics between $\mathrm{AMR}^{\mathrm{Pos}}$ and $\mathrm{AMR}^{\mathrm{Neg}} \mathrm{LT}$ patient populations. In our patient categorization, $\mathrm{AMR}^{\mathrm{Pos}}$ patients match the new consensus criteria of definite (C4d positive DSA positive) or probable (C4d negative DSA positive) clinical
AMR (12). Both immunodominant DSA MFI, sum MFI and DQ DSA MFI show high diagnostic performance for distinguishing between $\mathrm{AMR}^{\mathrm{Pos}}$ and $\mathrm{AMR}^{\mathrm{Neg}}$ patients. Moreover, DQ DSAs are associated with worse clinical outcomes through the occurrence of AMR.

Considering the cumulative DSA status of our cohort, 50\% of patients were DSA ${ }^{\text {Pos }}$. These results are in line with previous reports $(6,15,16)$. 


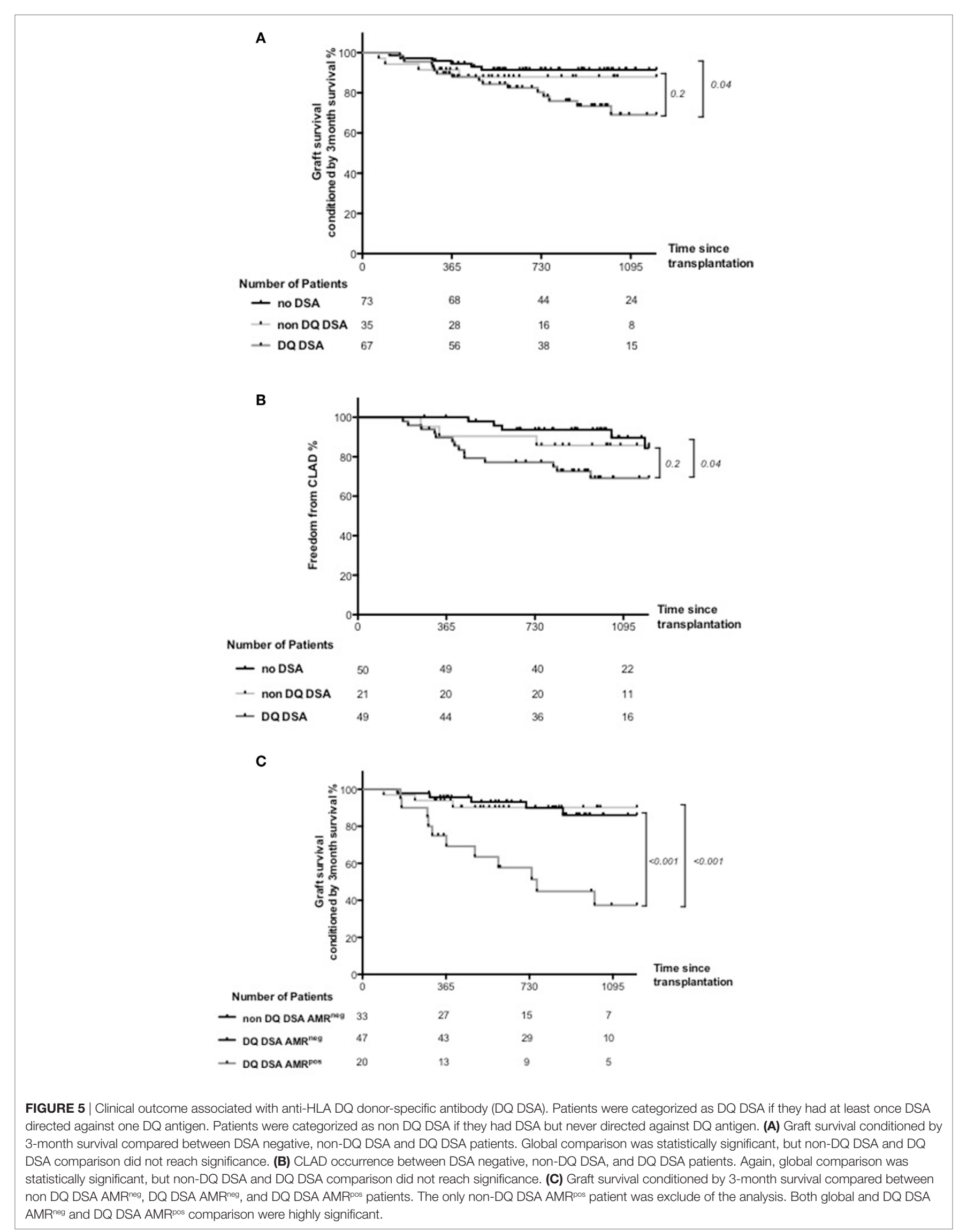


We found $\mathrm{AMR}^{\text {Pos }}$ patients to have significantly higher DSA MFI (either immunodominant or sum MFI) than their $\mathrm{AMR}^{\mathrm{Neg}}$ counterparts. The MFI values for $\mathrm{AMR}^{\mathrm{Pos}}$ patients in our cohort were similar to those described by Lobo et al. (7), but lower than those values found in KT studies (9). The lower MFI values reported in LT may be partly due to the "sponge effect" related to the higher capillary surface in the lung. Of note, DSA adsorption in the graft was first described decades ago (17), but the real impact of this phenomenon on DSA underestimation remains unclear. Futures studies analyzing intragraft DSA may bring new information about the reality of sponge effect in LT (18). Alternatively, there may be an organspecific susceptibility to DSA, due either to varying expression levels of complement inhibitory receptors and HLA molecules or differential responses to DSA ligation of HLA depending on the microvascular bed (19).

Sum MFI integrate the number of specificities for a given patient. Being aware that this summation only approximately represents the biological complexity of antibody-antigen interaction, in the case of multiple DSA, the sum MFI may be a more appropriate estimation of the DSA load than immunodominant DSA MFI alone.

In line with a previous publication (14), we also found that DQ DSAs were overrepresented in $\mathrm{AMR}^{\text {Pos }}$ patients. Not only, we demonstrate a specific DQ DSA effect on graft survival and CLAD, as Tikkanen did (11), but our results suggest also that these poor clinical outcomes were mainly associated with AMR occurrence.

Taken together, these results suggest (i) a specific association between DQ and AMR and (ii) that the negative impact of DQ DSA could be mainly driven by AMR occurrence. Consequently, whether DQ matching could prevent AMR occurrence warrants further investigation.

Importantly, AMR also occurred in the absence of DQ DSA indicating that DQ DSA is not a prerequisite for AMR. On the other hand, the fact that every DQ DSA does not necessarily lead to AMR emphasize our need for better characterization of pathogenic DSA. ROC evaluation suggests that the DSA MFI (sum MFI, immunodominant MFI, and DQ MFI) could help to identify $\mathrm{AMR}^{\mathrm{Pos}}$ and $\mathrm{AMR}^{\mathrm{Neg}}$ patients at the peak time point. To our knowledge, this is the first report of this kind.

However, the overlap between $\mathrm{AMR}^{\mathrm{Pos}}$ and $\mathrm{AMR}^{\mathrm{Neg}}$ patients was consistent, as $50 \%$ of the $\mathrm{AMR}^{\text {Pos }}$ patients and $40 \%$ of $\mathrm{AMR}^{\mathrm{Neg}}$ patients had immunodominant MFI between 2,100 and 10,000, and $68 \%$ of the $\mathrm{AMR}^{\mathrm{Pos}}$ patients and $47 \%$ of $\mathrm{AMR}^{\mathrm{Neg}}$ patients had a sum MFI between 2,100 and 10,000.

Besides the large overlap limitating the diagnosis value of MFI, either immunodominant or sum MFI show a very high sensitivity for AMR when considering a 10,000 cutoff.

In general, putative explanations for $\mathrm{AMR}^{\mathrm{Pos}}$ patients with a "low" MFI include the prozone effect (20), the aforementioned sponge effect, and concurrent undetected non-HLA DSA.

Altogether, these findings should be interpreted with caution and serve as an advocate for interpreting DSA results in the context of graft clinical status. In addition, single antigen tests performed with EDTA serum treatment to avoid the prozone effect, or titration, may be very helpful to enhance robustness of the assay. Finally, in cases where MFI values are neither "low" nor "high" (within the range of 2,000-10,000), complement activating potential or IgG subtypes of DSA (21) or intragraft DSA (18) assays may help to stratify risk of graft loss, and as such should be further explored.

The retrospective nature of our study is a limitation, although it did allow for the identification of the peak time point with sufficient followup. The peak time point was chosen for $\mathrm{AMR}^{\mathrm{Neg}}$ patients as the sample with the highest sum MFI value to allow for maximal alloantibody assault, and thereby the most appropriate to compare against $\mathrm{AMR}^{\mathrm{Pos}} \mathrm{MFI}$ values. This retrospective analysis will have to be prospectively validated, ideally in a multicentric study allowed by the recent ISHLT consensus for AMR diagnosis in LTx (12). Importantly, given the differences between the two single antigen test suppliers, our results only apply for the One Lambda ${ }^{\circledR}$ platform. Therefore, specific validation of this analysis should be also performed using the Immucor $^{\circledR}$ single antigen platform. Moreover, according to a $20 \%$ coefficient of variation allowance (22), MFI quantification deemed to be driven by antigen density at the surface of the bead, antibody:antigen affinity, and the prozone effect, should be interpreted using flexible thresholds. Lastly, current SAB tests only explore DSA directed against HLA, and a high-throughput detection tool for non-HLA DSA is not yet available to guide clinical care. This type of test would be particularly relevant in the case of an AMR clinical pattern with low MFI HLA DSA or without DSA (23).

\section{CONCLUSION}

We demonstrate that $\mathrm{AMR}^{\text {Pos }} \mathrm{LT}$ patients have higher number of specificities and increased MFI values when compared to $\mathrm{AMR}^{\mathrm{Neg}}$ patients. DQ DSAs were associated with poorer clinical outcomes through AMR occurrence.

Even so, given the wide distribution of MFI in both $\mathrm{AMR}^{\text {Pos }}$ and $\mathrm{AMR}^{\mathrm{Neg}}$ groups, DSA results should be interpreted with high regard for clinical graft failure status and additional DSA testing such as IgG subtype, complement binding capacity or intragraft DSA detection should be evaluated for a more accurate risk assessment for graft loss.

\section{THE FOCH LUNG TRANSPLANTATION GROUP}

Dr Clément Picard, Dr. Dominique Grenet, Dr. Sandra De Miranda, Dr. Abdul Monem Hamid, Dr. Benoit Douvry, Dr. Laurence Beaumont-Azuar, Dr. Geneviève Le Bourdelles, Dr. Hélène Neveu, Dr. Daniela Usturoi, Dr. Marc Stern, Dr. Antoine Roux; Pneumology Department, Foch Hospital, Suresnes France; Dr. Alain Chapelier, Dr. Edouard Sage, Dr. Philippe Puyo, Dr. Pierre Bonnette, Dr. Jocelyn Bellier, Dr. Mathieu Glorion, Dr. Salam AbouTaam, Dr. François Gonin, Dr. Triet Ngo, Thoracic Surgery Department, Foch Hospital, Suresnes France; Dr. Elise 
Cuquemelle, Dr. François Parquin, Thoracic Intensive Care Unit, Foch Hospital, Suresnes France; Dr Charles Cerf, Dr. Grégoire Trebbia, Dr. Alexis Soummer, Dr. Jêrome Devaquet, Dr. Anthony Lanceleur, Dr. Koceila Bouferrache, Dr. David Courtier, Dr. Vincent Caille, Dr. Anne-Gaëlle Si Larbi, Intensive Care Unit, Foch Hospital, Suresnes France; Dr. Marc Fischler, Dr. Morgan Le Guen, Dr. Virginie Dumans-Nizard, Dr. Barbara Szekely, Dr. Mireille Michel-Cherqui, Dr. Jean- Yves Marandon, Dr. Ngai Liu, Dr. Léa Ley, Dr. Julie Bresson, Dr. Marie Louise Felten, Dr. Béatrice Angemont, Dr. Valentina Assenzo, Dr. Olivier Belze, Dr. Marie Binczak, Dr. Patrick Clapson, Dr. Camille Cornet, Dr. Jean-Louis Dumoulin, Dr. Sébastien Jacqmin, Dr. Olivier Pruszkowski, Dr. Adrian Radu, Dr. Céline Roussel, Dr. Benoît Vandenbunder, Dr. Nicolas Verroust, Dr. Thibaut Mariaux de Serres, Anesthesiology Department, Foch Hospital, Suresnes France; Dr. Leila Zemoura; Dr. Yves Denoux; Sonia Holifanjaniaina; Dr. Elisabeth Longchampt; Dr. Jean-Marc Guinebretiére, Pathology Department, Foch Hospital, Suresnes France; Dr François Mellot; Dr. Axel Guth; Dr. Nadia Canepa, Radiology Department, Foch Hospital, Suresnes France; Dr. Marc Vasse, Dr. Eric Farfour, Dr. Emilie Cardot, Dr. Pierre Cahen, Dr. Philippe Lesprit, Dr. Damien Mathonnet, Biology Department, Foch Hospital, Suresnes France; Dr Sophie Hillaire, Dr. Christine Veyrie, Dr. Florence Bouilloud, Dr. Barbara Néraud, Internal Medecine, Foch Hospital, Suresnes France; Dr. Dominique Dardelle, Dr. Eve Camps, Pharmacy Department, Foch Hospital, Suresnes France.

\section{REFERENCES}

1. Einecke G, Sis B, Reeve J, Mengel M, Campbell PM, Hidalgo LG, et al. Antibody-mediated microcirculation injury is the major cause of late kidney transplant failure. Am J Transplant (2009) 9:2520-31. doi:10.1111/j.1600-6143.2009.02799.x

2. Haas M, Sis B, Racusen LC, Solez K, Glotz D, Colvin RB, et al. Banff 2013 meeting report: inclusion of $\mathrm{c} 4 \mathrm{~d}$-negative antibody-mediated rejection and antibody-associated arterial lesions. Am J Transplant (2014) 14:272-83. doi:10.1111/ajt.12590

3. Sellarés J, de Freitas DG, Mengel M, Reeve J, Einecke G, Sis B, et al. Understanding the causes of kidney transplant failure: the dominant role of antibody-mediated rejection and nonadherence. Am J Transplant (2012) 12:388-99. doi:10.1111/j.1600-6143.2011.03840.x

4. Kfoury AG, Snow GL, Budge D, Alharethi RA, Stehlik J, Everitt MD, et al. A longitudinal study of the course of asymptomatic antibody-mediated rejection in heart transplantation. J Heart Lung Transplant (2012) 31:46-51. doi:10.1016/j.healun.2011.10.009

5. Roux A, Bendib Le Lan I, Holifanjaniaina S, Thomas KA, Hamid AM, Picard C, et al. Antibody-mediated rejection in lung transplantation: clinical outcomes and donor-specific antibody characteristics. Am J Transplant (2016) 16(4):1216-28. doi:10.1111/ajt.13589

6. Morrell MR, Pilewski JM, Gries CJ, Pipeling MR, Crespo MM, Ensor CR, et al. De novo donor-specific HLA antibodies are associated with early and highgrade bronchiolitis obliterans syndrome and death after lung transplantation. J Heart Lung Transplant (2014) 33:1288-94. doi:10.1016/j.healun.2014.07.018

7. Lobo LJ, Aris RM, Schmitz J, Neuringer IP. Donor-specific antibodies are associated with antibody-mediated rejection, acute cellular rejection, bronchiolitis obliterans syndrome, and cystic fibrosis after lung transplantation. J Heart Lung Transplant (2013) 32:70-7. doi:10.1016/j.healun.2012.10.007

\section{ETHICS STATEMENT}

This observational study was approved by the research protocol evaluation committee of the Institutional Review Board of the French Learned Society for Respiratory Medicine-Sociéte de Pneumologie de Langue Française. Every patient in this study was enrolled in a prospective non-therapeutic interventional research protocol [eitherCOLT(NCT00980967,ID-RCB:2009-A00036-51) or RhumTP (NCT01791166, ID-RCB:2010-A01174-35)]. Upon enrollment, all patients signed consent for research use of their clinical data.

\section{AUTHOR CONTRIBUTIONS}

Research design: AR, MS, FP, CS, and KT. Writing of the paper: AR, MS, FP, CS, and KT. Performance of the research: AR, MS, FP, CS, CG, IL, SH, CP, DG, SM, BD, LB-A, ES, JD, EC, MG, and AH. Analytic tools: MR and KT. Data analysis: AR, MS, FP, CS, $\mathrm{KT}$, and IL.

\section{ACKNOWLEDGMENTS}

The authors thank N. M. Valenzuela, E. F. Reed, and R. Spreafico for critical review of the manuscript.

\section{FUNDING}

This work was supported by the "Fondation Foch," the "Association Gregory Lemarchal," and the "Fondation du Souffle."

8. Malheiro J, Tafulo S, Dias L, Martins LS, Fonseca I, Beirão I, et al. Analysis of preformed donor-specific anti-HLA antibodies characteristics for prediction of antibody-mediated rejection in kidney transplantation. Transpl Immunol (2015) 32:66-71. doi:10.1016/j.trim.2015.01.002

9. Mujtaba MA, Goggins W, Lobashevsky A, Sharfuddin AA, Yaqub MS, Mishler DP, et al. The strength of donor-specific antibody is a more reliable predictor of antibody-mediated rejection than flow cytometry crossmatch analysis in desensitized kidney recipients. Clin Transplant (2011) 25:E96-102. doi:10.1111/j.1399-0012.2010.01341.x

10. Yell M, Muth BL, Kaufman DB, Djamali A, Ellis TM. C1q binding activity of de novo donor-specific HLA antibodies in renal transplant recipients with and without antibody-mediated rejection. Transplantation (2015) 99:1151-5. doi:10.1097/TP.0000000000000699

11. Tikkanen JM, Singer LG, Kim SJ, Li Y, Binnie M, Chaparro C, et al. De novo DQ-donor-specific antibodies are associated with chronic lung allograft dysfunction after lung transplantation. Am J Respir Crit Care Med (2016) 194(5):596-606. doi:10.1164/rccm.201509-1857OC

12. Levine DJ, Glanville AR, Aboyoun C, Belperio J, Benden C, Berry GJ, et al. Antibody-mediated rejection of the lung: a consensus report of the International Society for Heart and Lung Transplantation. J Heart Lung Transplant (2016) 35:397-406. doi:10.1016/j.healun.2016.01.1223

13. Verleden GM, Raghu G, Meyer KC, Glanville AR, Corris P. A new classification system for chronic lung allograft dysfunction. J Heart Lung Transplant (2014) 33:127-33. doi:10.1016/j.healun.2014.01.316

14. Tagliamacco A, Cioni M, Comoli P, Ramondetta M, Brambilla C, Trivelli A, et al. DQ molecules are the principal stimulators of de novo donor-specific antibodies in nonsensitized pediatric recipients receiving a first kidney transplant. Transpl Int (2014) 27:667-73. doi:10.1111/tri.12316

15. Hachem RR, Yusen RD, Meyers BF, Aloush AA, Mohanakumar T, Patterson GA, et al. Anti-human leukocyte antigen antibodies and preemptive 
antibody-directed therapy after lung transplantation. J Heart Lung Transplant (2010) 29:973-80. doi:10.1016/j.healun.2010.05.006

16. Safavi S, Robinson DR, Soresi S, Carby M, Smith JD. De novo donor HLAspecific antibodies predict development of bronchiolitis obliterans syndrome after lung transplantation. J Heart Lung Transplant (2014) 33:1273-81. doi:10.1016/j.healun.2014.07.012

17. Frost AE, Jammal CT, Cagle PT. Hyperacute rejection following lung transplantation. Chest (1996) 110:559-62. doi:10.1378/chest.110.2.559

18. Visentin J, Chartier A, Massara L, Linares G, Guidicelli G, Blanchard E, et al. Lung intragraft donor-specific antibodies as a risk factor for graft loss. J Heart Lung Transplant (2016) 35(12):1418-26. doi:10.1016/j.healun.2016.06.010

19. Valenzuela NM, Reed EF. Antibodies in transplantation: the effects of HLA and non-HLA antibody binding and mechanisms of injury. Methods Mol Biol (2013) 1034:41-70. doi:10.1007/978-1-62703-493-7_2

20. Guidicelli G, Anies G, Bachelet T, Dubois V, Moreau JF, Merville P, et al. The complement interference phenomenon as a cause for sharp fluctuations of serum anti-HLA antibody strength in kidney transplant patients. Transpl Immunol (2013) 29:17-21. doi:10.1016/j.trim.2013.09.005

21. Lefaucheur C, Viglietti D, Bentlejewski C, Duong van Huyen JP, Vernerey D, Aubert $\mathrm{O}$, et al. IgG donor-specific Anti-human HLA antibody subclasses and kidney allograft antibody-mediated injury. JAm Soc Nephrol (2016) 27:293-304. doi:10.1681/ASN.2014111120
22. Reed EF, Rao P, Zhang Z, Gebel H, Bray RA, Guleria I, et al. Comprehensive assessment and standardization of solid phase multiplex-bead arrays for the detection of antibodies to HLA. Am J Transplant (2013) 13:1859-70. doi:10.1111/ajt.12287

23. Reinsmoen NL, Lai CH, Heidecke H, Haas M, Cao K, Ong G, et al. Antiangiotensin type 1 receptor antibodies associated with antibody mediated rejection in donor HLA antibody negative patients. Transplantation (2010) 90:1473-7. doi:10.1097/TP.0b013e3181fd97f1

Conflict of Interest Statement: AR has conflicts of interest to disclose as described by the Transplant International journal: he served as a consultant for Novartis France (concerning CMV in solid organ transplantation). The other authors have no conflicts of interest to disclose.

Copyright (๑) 2017 Roux, Bendib Le Lan, Holifanjaniaina, Thomas, Picard, Grenet, De Miranda, Douvry, Beaumont-Azuar, Sage, Devaquet, Cuquemelle, Le Guen, Suberbielle, Gautreau, Stern, Rossetti, Hamid and Parquin. This is an open-access article distributed under the terms of the Creative Commons Attribution License (CC BY). The use, distribution or reproduction in other forums is permitted, provided the original author(s) or licensor are credited and that the original publication in this journal is cited, in accordance with accepted academic practice. No use, distribution or reproduction is permitted which does not comply with these terms. 